\title{
Our experience with an extremely rare condition of impacted "Kissing Molars"
}

\author{
Atanaska Cheshmedzhieva ${ }^{1}$, Slavi Nachev ${ }^{1}$, Rosen \\ Kolarov $^{2}$, Stanislav Kolev ${ }^{1}$
}

1. Department of Oral surgery, Military Medical Academy, Sofia, Bulgaria;

2. National Medical Coordination Center, Ministry of Health, Sofia, Bulgaria;

\begin{abstract}
"Kissing molars" (KM) or "rosette formation" is a term used to describe impacted teeth with contacting occlusal surfaces in a single follicular space and their roots pointing in opposite directions. Kissing molars can be observed rarely in some patients but occurrence of bilateral kissing molars is an extremely rare phenomenon in dental literature and its aetiology is still unknown. In this paper we describe our experience with this pathology for a period of 10 years. In our case, an extremely rare condition of impacted bilateral kissing molars was resolved by means of surgical extraction. The decision-making for extraction of the asymptomatic kissing molars represents a surgical dilemma. Such cases may present with a risk of numerous surgical complications while in many of them surgical intervention is unavoidable. Few treatment options are described in the literature. This phenomenon can be a sign of various medical conditions that may require further investigation. In this paper our treatment option responds to the literature suggesting surgical removal of both impacted molars at either side of the mandible.
\end{abstract}

Keywords: Impacted teeth, Kissing molars, Surgical removal; 


\section{Introduction}

Impaction problems primarily affect permanent teeth with mandibular and maxillary third molars being most frequent, followed by maxillary canines, central incisors, mandibular second premolars and less frequently second molars $(0.03-0.04 \%$ of all affected teeth, $(1,12,14))$. The term "kissing molars" or "rosette formation" is used to describe impacted second and third molars and supernumerary teeth in rare instances, with contacting occlusal surfaces within a single dental follicle and with roots directed in opposite directions $(2,3)$. This term has also been used to describe similar conditions affecting other teeth(7). This type of impaction was first described by Van Hoof in 1973. In some cases bilateral kissing molars in both jaws can be observed. Being an extremely rare phenomenon, the etiology of such condition remains unexplored(8).

\section{Case report:}

In the current article three clinical cases of kissing molars are presented. Those we encountered in a period of 10 years in our hospital. All three patients were in their twenties - one male and two females. All of them came to the Department of Oral and Maxillofacial Surgery complaining basically of swelling in the area of impaction and irradiating pain in the adjacent teeth. Intraoral inspection revealed expansion of the vestibular cortical plate and a soft tumefaction overlaying the residual alveolar process in the area. Medical history of the patients was non-relevant to good overall health status, no allergies or medical conditions and no prescribed medicines. The patients provided previously done panoramic radiographs. A CT scan was deemed unnecessary. The x-rays revealed impaction of second and third molars on either side of the mandible and in the maxillary tuberosities (Fig. 1 a,b,c).

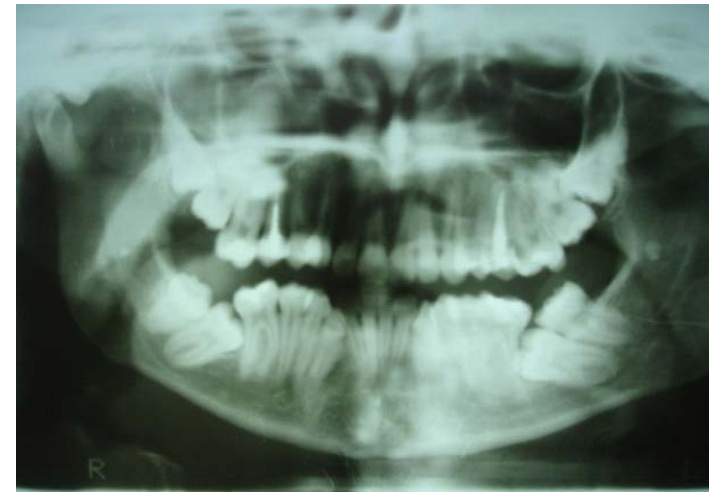

Figure 1a

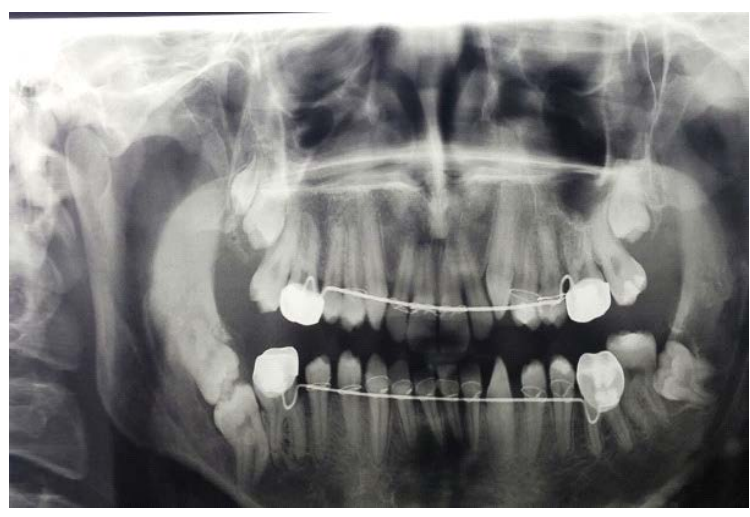

Figure 1b 


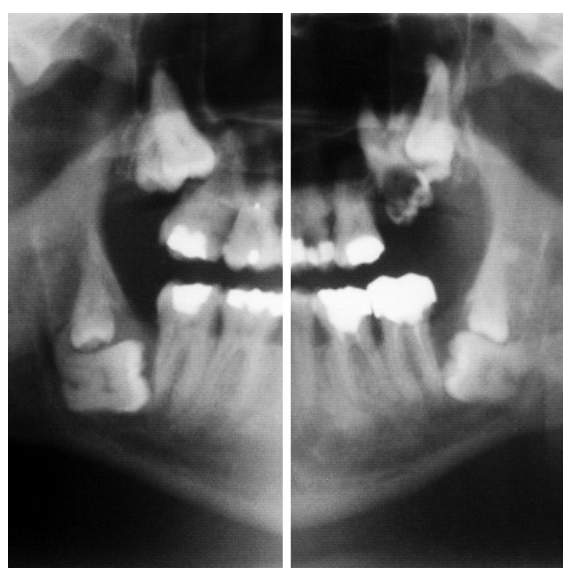

Figure 1c

Our treatment plan was made up of a surgical removal of the "kissing molars"(4,13). A thorough preoperative medical assessment was carried out, including blood tests. The latter showed no health problems. Risk of complications was ranked low(15). Surgery procedures in all three patients were performed under mandibular-nerve-block local anesthesia. $40 \mathrm{mg}$ of intramuscular methylprednisolon was administered pre-operatively to lower the risk of post-operative events. N15 blade was used to make a bayonet incision in the area of the "kissing molars" and a vestibular muco-periosteal flap was raised. After that osteotomy was performed to reveal the crowns of the "kissing" teeth. Next step was a cervical sectioning in both molars which contributed to the removal of less bone and facilitated the procedure. After the removal of the third molar, the second one was extracted in the same manner. Following teeth extraction a saline irrigation and removal of the residual follicle were performed (Fig.2,3,4,5). Finalization was accomplished through clindamycin solution application in the surgical wound, placing a hemostatic matrix and a $3 / 0$ interrupted silk suture.

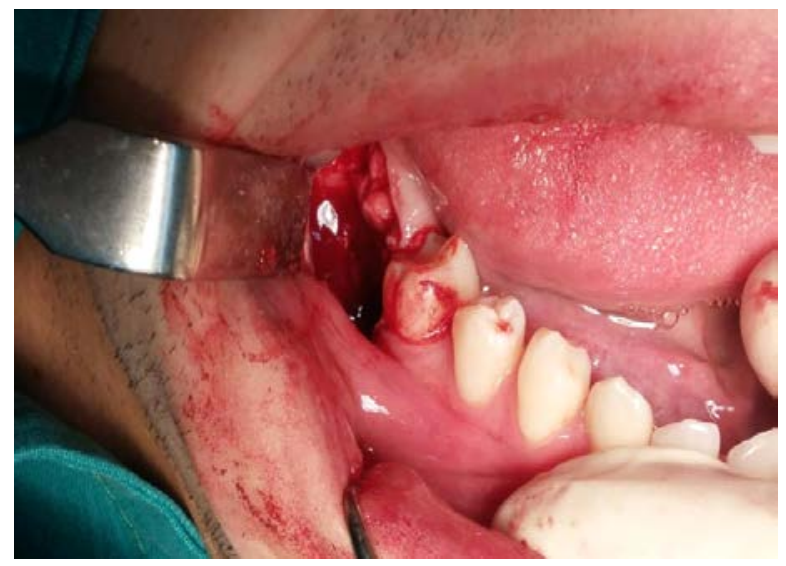

Figure 2

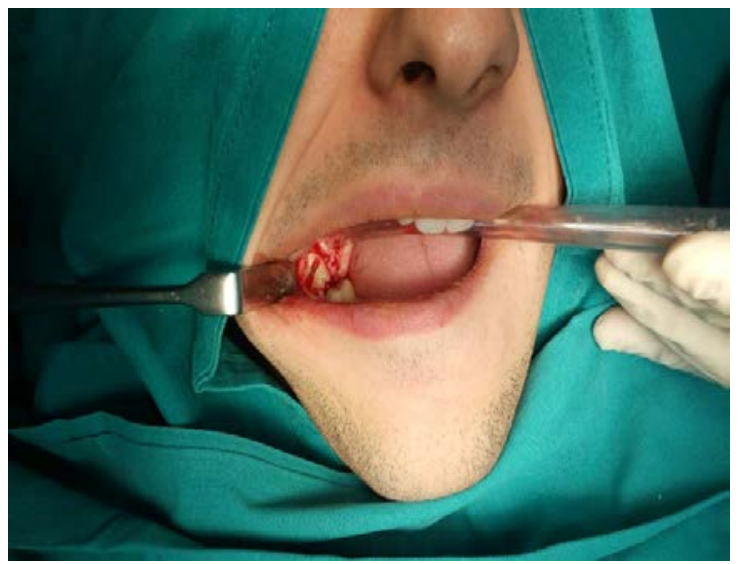

Figure 3 


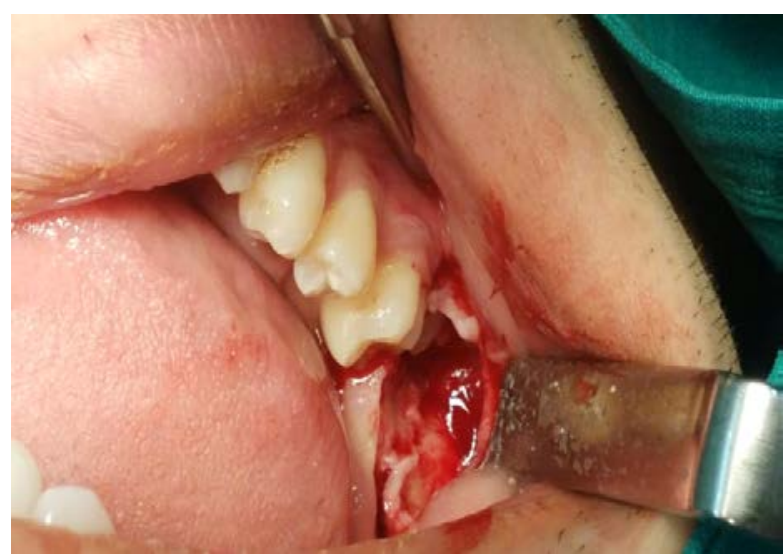

Figure 4

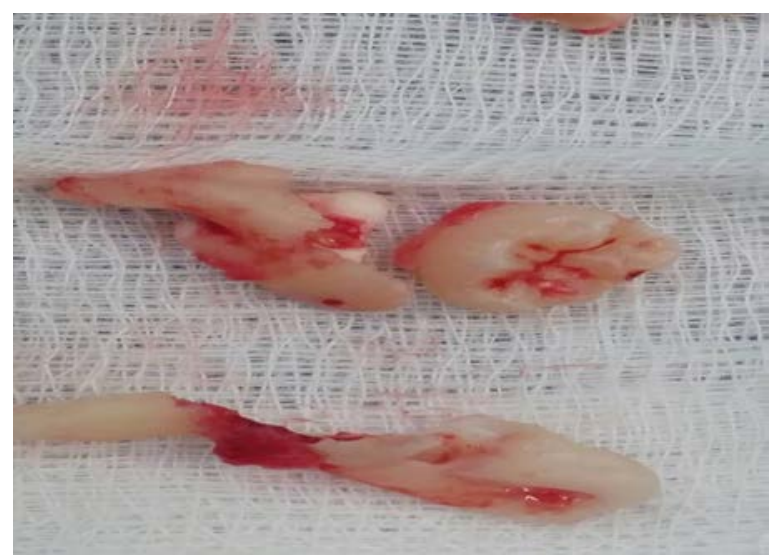

Figure 5

A three-month post-operative period was allowed before proceeding to further surgical procedures in the rest indicated impacted areas in each patient. A dose of $900 \mathrm{mg}$ a day "Dalacin C" antibiotic was prescribed for a period of one week post-operatively to serve minimizing pain and infection. The intra-oral swelling shrank gradually in the follow-up period $(5,6,17)$. Patients were advised on a liquid diet for one week after surgery and a further soft food diet for over a month and a half. No post-operative dental, soft tissue or jaw sensitivity changes were detected. Evolution of other common post-extraction complications was not observed.

\section{Discussion:}

"Kissing molars" or "rosette formation" is a variety of dental impaction rarely reported in literature $(4,11)$. Our review confirmed an original report of such a case in 1973(1,3). It referred to second and third mandibular molars positioned in one dental follicle, the occlusal surfaces of which contacted each other and the roots pointing in opposite directions. We have come across only three such cases in our department for the last decade. These make up 0.08 percent of all impacted teeth treated in our theatre. The aetiology of this condition is yet to be uncovered $(1,16)$. There are reports in literature of multiple "rosette formations" or 'bilateral "kissing molars" associated with mucopolysaccharidosis (MPS, $(9,10)$ ). The latter is a group of inherited metabolic disorders brought about by an alteration in depositing specific enzymes in different body tissues. The dental condition reported by our team in this article could be connected to MPS, although no scientific explanation has been given yet. Cawson reports a case with MPS and suggests that the disorder could play a role as a possible aetiology factor for "kissing molars". Furthemore, Nakamura reports MPS in two out of four patients in his study. Opting to manage "kissing molars" through operative extraction is a surgical conundrum as many complications could ensue, including mandibular fracture during or after removal, alveolitis, inferior alveolar nerve ( 0.5 to 5.0 percent) or lingual nerve (0.2 to 2.0 percent) impairment, osteomyelitis or damage to TMJ. However, retaining the "kissing molars" could also lead to other problems such as bone loss, increased risk of mandibular 
fracture, pericoronitis, localized pain, cystic lesions or root resorption in adjacent teeth. In favor of lessening and staying away from such complications we recommend a surgical approach. Panoramic $\mathrm{x}-$ rays are the gold standart in most cases. CT scan can be used to identify precisely nearby vital structures as well as the mandibular canal and the lingual nerve, thus avoiding injury..

\section{Conclusion}

Dentists encounter various kinds of tooth impaction in their practice. "Kissing molars" is an extremly rare example and sufficient information describing it still lacks. Few treatment options can be found in literature. There is data to suggest that this condition could be a mark in various medical disorders, necessitating further investigation. In the current article our treatment option coresponds entirely to literature factology and states a surgical removal of all impacted teeth.

\section{References}

1. Stefano S., Michela R., Paolo B., et al. Chapter11: uprighting of the impacted second mandibular molar with skeletal anchorage. In: Bourzgui F., editor. Orthodontics Basic Aspects and Clinical Considerations. 2012. pp. 247-264.

2. Yadavalli G., Singh C. D. Kissing molars-a rare entity. Journal of Pharmaceutical and Biomedical Sciences. 2013;3:1245-1246.

3. van Hoof R. F. Four kissing molars. Oral Surgery, Oral Medicine, Oral Pathology. 1973;35(2, article 284) doi: 10.1016/0030-4220(73)90297-1.

4. Bakaeen G., Baqain Z. H. Interesting case: kissing molars. British Journal of Oral and Maxillofacial Surgery. 2005;43(6):p. 534. doi: 10.1016/j.bjoms.2004.10.020.

5. Adrian Robinson J., Gaffney W., Jr., Soni N. N. Bilateral 'kissing' molars. Oral Surgery, Oral Medicine, Oral Pathology. 1991;72(6, article 760) doi: 10.1016/0030-4220(91)90026-9.

6. Krishnan B. Kissing molars. British Dental Journal. 2008;204(6):281-282. doi: 10.1038/bdj.2008.216.

7. Juneja M. Not kissing. British Dental Journal. 2008;204(11):p. 597. doi: 10.1038/sj.bdj.2008.470.

8. McIntyre G. Kissing molars: an unexpected finding. Dental Update. 1997;24(9):373-374.

9. Graupman P., Pan D., Konair B., et al. Craniofacial abnormalities in a murine knock-out model of mucopolysaccharidosis I H: a computed tomography and anatomic study. The Journal of Craniofacial Surgery.2004;15(3):392-398. doi: 10.1097/00001665-200405000-00009.

10. 10. Cawson R. A. The oral changes in gargoylism. Proceedings of the Royal Society of Medicine.1962;55:1066-1070.

11. Nakamura T., Miwa K., Kanda S., et al. Rosette formation of impacted molar teeth in mucopolysaccharidoses and related disorders. Dentomaxillofacial Radiology. 1992;21(1):45-49. doi: 10.1259/dmfr.21.1.1397452.

12. McCoy J. M. Complications of retention: pathology associated with retained third molars. Atlas of the Oral and Maxillofacial Surgery Clinics of North America. 2012;20(2):177-195. doi: 10.1016/j.cxom.2012.06.002

13. Giraldi Neto F. O., Rocha Júnior H. V., Martinez Júnior W., Duarte B. G., Salgueiro D. G., Sant'ana E. Interesting cases of kissing molars. Report of two cases. Revista de Odontologia da UNESP. 2012;41(4):292-295. 
14. Gulses A., Varol A., Sencimen M., Dumlu A. A study of impacted love: kissing molars. Oral Health and Dental Management. 2012;11(4):185-188.

15. Robinson J. A., Gaffney W., Jr., Soni N. N. Bilateral 'kissing' molars. Oral Surgery, Oral Medicine, Oral Pathology. 1991;72(6):p. 760. doi: 10.1016/0030-4220(91)90026-9.

16. Bakaeen G., Baqain Z. H. İtersting case: kissing molars. British Journal of Oral and Maxillofacial Surgery. 2005;43(6):p. 534. doi: 10.1016/j.bjoms.2004.10.020.

17. Cheshmedzhieva A.: Application of Platelet-rich plasma in oral surgery cases, Thesis, 2015

\section{Corresponding author:}

Atanaska Cheshmedzhieva DDS, PhD

Tel: +359 886899 599;

e-mail: acheshmedzhieva@gmail.com 